\title{
Alzheimer disease in African American individuals: increased incidence or not enough data?
}

\section{Lisa L. Barnes}

\begin{abstract}
Research on racial differences in Alzheimer disease (AD) dementia has increased in recent years. Older African American individuals bear a disproportionate burden of $A D$ and cognitive impairment compared with non-Latino white individuals. Tremendous progress has been made over the past two decades in our understanding of the neurobiological substrates of AD. However, owing to well-documented challenges of study participant recruitment and a persistent lack of biological data in the African American population, knowledge of the drivers of these racial disparities has lagged behind. Therapeutic targets and effective interventions for $A D$ are increasingly sought, but without a better understanding of the disease in African American individuals, any identified treatments and/or cures will evade this rapidly growing at-risk population. In this Perspective, I introduce three key obstacles to progress in understanding racial differences in AD: uncertainty about diagnostic criteria, disparate cross-sectional and longitudinal findings; and a dearth of neuropathological data. I also highlight evidence-informed strategies to move the field forward.
\end{abstract}

Today, one in eight older Americans ( $>65$ years of age) are affected by Alzheimer disease $(\mathrm{AD})$ dementia and related dementias, at an annual cost of more than US $\$ 200$ billion ${ }^{1}$. With future population increases in the oldest age groups, estimates indicate that 13.5 million individuals in the USA will have AD by the year 2050 . Thus, prevention of $A D$ is a public health priority. The most at-risk population in the USA is also becoming increasingly racially and ethnically diverse ${ }^{2}$, and a growing body of evidence suggests that older African American individuals bear a disproportionate burden of cognitive impairment and dementia compared with other racial and ethnic groups ${ }^{3-5}$. According to some estimates, the incidence of $\mathrm{AD}$ is almost twofold higher in older African American individuals than in older individuals of other minority racial and ethnic groups in the $\mathrm{USA}^{1-4}$; however, the reasons for the increased burden are unknown. African American individuals are under-represented in $\mathrm{AD}$ clinical research studies, particularly longitudinal studies that include racially relevant risk factors that reflect their lived experience and studies that include biomarkers and neuropathology. Therefore, the contributions of risk factors and disease pathologies to $\mathrm{AD}$ in this population are still poorly understood.

Throughout this perspective, the terms race and ethnicity are used. Both terms describe social constructs with no basis in biology, but in the medical literature, race is typically used to group people who are similar in ancestral background and/or share phenotypic features like skin colour. For example, in the US Census, five categories of race are officially recognized: white, Black or African American, Asian American, American Indian/Alaska Native, and Native Hawaiian/Pacific Islander. In contrast, ethnicity is used to group people who share common cultural traditions, languages and values regardless of racial classification (for example, people with cultural ties to Latin America are often referred to as being of Latino, Latina or Latinx ethnicity but can be of any race). In this Perspective, I specifically address the situation in the USA and use the term African American to refer to individuals who would be grouped by the US Census into the Black or African American category.

The ageing and dementia research community has identified a number of genetic, medical, and lifestyle factors that are associated with the risk of dementia. Although this knowledge, largely gathered from study cohorts that are overwhelmingly white, is generally assumed to characterize risk in all populations, we know very little about the drivers of disease in African American individuals. The preponderance of data from one population can lead to the false and potentially dangerous conclusion that that group represents some type of scientific norm that all other groups should be compared to. However, research on the drivers of $\mathrm{AD}$ in minoritized populations - that is, populations that have been systematically marginalized in society - is as important as research of drivers in the majority population, and should be the focus of studies, even if it does not explicitly inform us about racial differences.

The current evidence for higher rates of dementia among African American individuals is broad and has been comprehensively reviewed elsewhere ${ }^{4,6,7}$. It is generally well-accepted that in the USA race is a social construct based primarily on phenotypic traits that shape access to power and social and economic resources ${ }^{8}$. The effects of power differentials and social disadvantage might apply to other marginalized groups (for example, white individuals of low socioeconomic status, rural Americans, sexual and gender minorities, other racial or ethnic minorities, immigrants and people with disabilities); however, recent events related to the global coronavirus disease 2019 (COVID-19) pandemic, the public murder of George Floyd, and other instances of social unrest for African American people have made it clear that the $\mathrm{AD}$ disparities for this population in particular, have roots in structural and social determinants.

Despite the central role of genetics in $\mathrm{AD}$, racial disparities in $\mathrm{AD}$ are not completely explained by genetics. Indeed, reports have confirmed that $\mathrm{AD}$-related genetic variants found in white individuals also exist in African American individuals, 
although disease-associated loci within the common pathways involved in AD aetiology (for example, immunity, lipid processing, intracellular trafficking) and the risk attributable to the loci might differ ${ }^{10-12}$. Social factors linked to race ${ }^{13}$, prevalent vascular disease ${ }^{6}$, disparities in years and quality of education ${ }^{7}$, and unequal access to and/or inequitable use of health care ${ }^{14}$ are increasingly recognized as having important roles in the excess risk of $\mathrm{AD}$ in African American individuals, even if they do not completely account for the disparities.

Ultimately, the disproportionate burden of $\mathrm{AD}$ in the African American population is likely to be caused by a complex interaction of socially patterned environmental exposures (that is, the social exposome) and biological factors that accumulate throughout the life course. The challenge for those of us working in the field will be to address the methodological barriers that hamper our progress in studies of racial differences, and to become more integrative in our study designs and approaches, bringing to bear the interdisciplinary talent and expertise that is needed to unravel the complexity of the disease.

\section{Understanding racial differences}

At least two scientific arguments exist for studying racial differences in AD. First, understanding why risk factors for $\mathrm{AD}$ in African American individuals differ from those in white individuals will help make the accumulated research on white individuals relevant for African American individuals. Second, understanding these differences might help us learn new information about $\mathrm{AD}$ overall, including information that could improve diagnosis and prognosis, and open the door for further scientific discovery. However, major obstacles to understanding racial differences in $\mathrm{AD}$ exist - some methodological in nature and others reflecting an extreme lack of data.

Neuropsychological test performance. One important obstacle involves the current standard of making the diagnosis. A clinical diagnosis of $\mathrm{AD}$ requires cognitive impairment in at least two domains of cognition, as assessed by standard neuropsychological tests, and severe enough loss of function to interfere with daily activities $^{15}$. This threshold for diagnosis was developed in white older adults with the disease and is somewhat arbitrary for everyone regardless of race. However, substantial racial disparities are observed on standard neuropsychological tests, with older African American individuals tending to perform more poorly on a wide range of tests - particularly tests of executive function and visuospatial ability ${ }^{16,17}$ - than older white individuals, even when matched for level of education ${ }^{18}$. Disproportionate performance below the diagnostic threshold on these tests among African American individuals is likely to contribute to higher clinical incidence of dementia in this population. The degree of correspondence between test performance (or clinical diagnosis) and the underlying pathology that causes dementia can be quite variable ${ }^{19}$, possibly even more so for African American individuals ${ }^{20}$, although the data addressing this question are limited. However, even beyond the lack of correspondence between clinical symptoms and pathology, an emerging body of research brings into question whether these racial differences in test performance actually indicate a higher incidence of $\mathrm{AD}$ in African American individuals than other ethnic or racial groups or whether another explanation exists.

First, as documented in numerous studies, educational and cultural experiences often bias neuropsychological test performance ${ }^{21-24}$. To circumvent this problem, clinicians and/or researchers sometimes rely on the use of race-based norms, which are normative standards for neuropsychological tests that are adjusted according to race. These norms are typically created by testing a large representative sample of people from the same racial group (either nationally representative or representative of the local setting) who vary along a range of characteristics that are important for neuropsychological tests such as years of education, and are used to judge and interpret test performance for that particular racial group. However, although race-based norms are helpful for increasing diagnostic accuracy in clinical settings, their use makes a number of assumptions about biological constructions of race that might lead to erroneous and potentially harmful interpretations of underlying racial differences ${ }^{24}$. Furthermore, use of these norms makes it virtually impossible to understand why such differences exist in the first place. The other strategy for dealing with biases in test performance is to adjust for factors that might influence performance in cross-sectional assessments. However, even when adjustments are made for the factors that make the measures less valid in African American individuals (for example, years of education or socioeconomic status), a host of other factors can influence test performance among African American individuals and are not typically considered when interpreting levels of cognitive impairment ${ }^{25-27}$, especially in studies that compare African American individuals to white individuals ${ }^{28,29}$. For example, educational quality has been known for some time to be a stronger predictor of test performance among African American individuals than years of education ${ }^{30,31}$; a finding that has been consistently replicated over the years ${ }^{32}$. However, the field has struggled to document robust measures of education quality and such measures are rarely used to facilitate the interpretation of test results in the clinical context ${ }^{33}$.

Interestingly, many of the factors that influence test performance in African American individuals, such as quality of education, and experiences of early-life educational segregation and discrimination, often seem to affect initial neuropsychological test performance but not cognitive decline. For example, a study published in 2019 (REF. $^{25}$ ) found that African American individuals born in a southern US state (or living in that state at age 12 years) as determined by US Census geographic designations, performed at lower levels across five domains of cognitive performance than individuals born or living in a northern US state at age 12 years. State of birth also interacted with school segregation experience such that participants who lived in the South and attended a legally desegregated school had the lowest performance of any group, including participants who lived in the South and attended a segregated school or those who lived in the North and attended either type of school. Neither factor, state of birth or segregation experience, was related to cognitive decline; only baseline level of performance. These, and similar findings are consistent with the idea that factors particularly reflective of historical experiences of marginalized populations in the USA have an important negative impact on test performance and might contribute to claims of an increased risk of dementia in these populations.

Study design. Another obstacle is the discrepancy between the racial differences in $\mathrm{AD}$ observed in cross-sectional studies and those observed in longitudinal studies (TABLE 1). Most of the existing literature on race and cognition comes from cross-sectional studies, in which cognition is measured at one point in time and compared with that in a white comparison group. Such studies have 
Table 1 | Studies of age-related cognitive decline in Black or African American individuals and white individuals

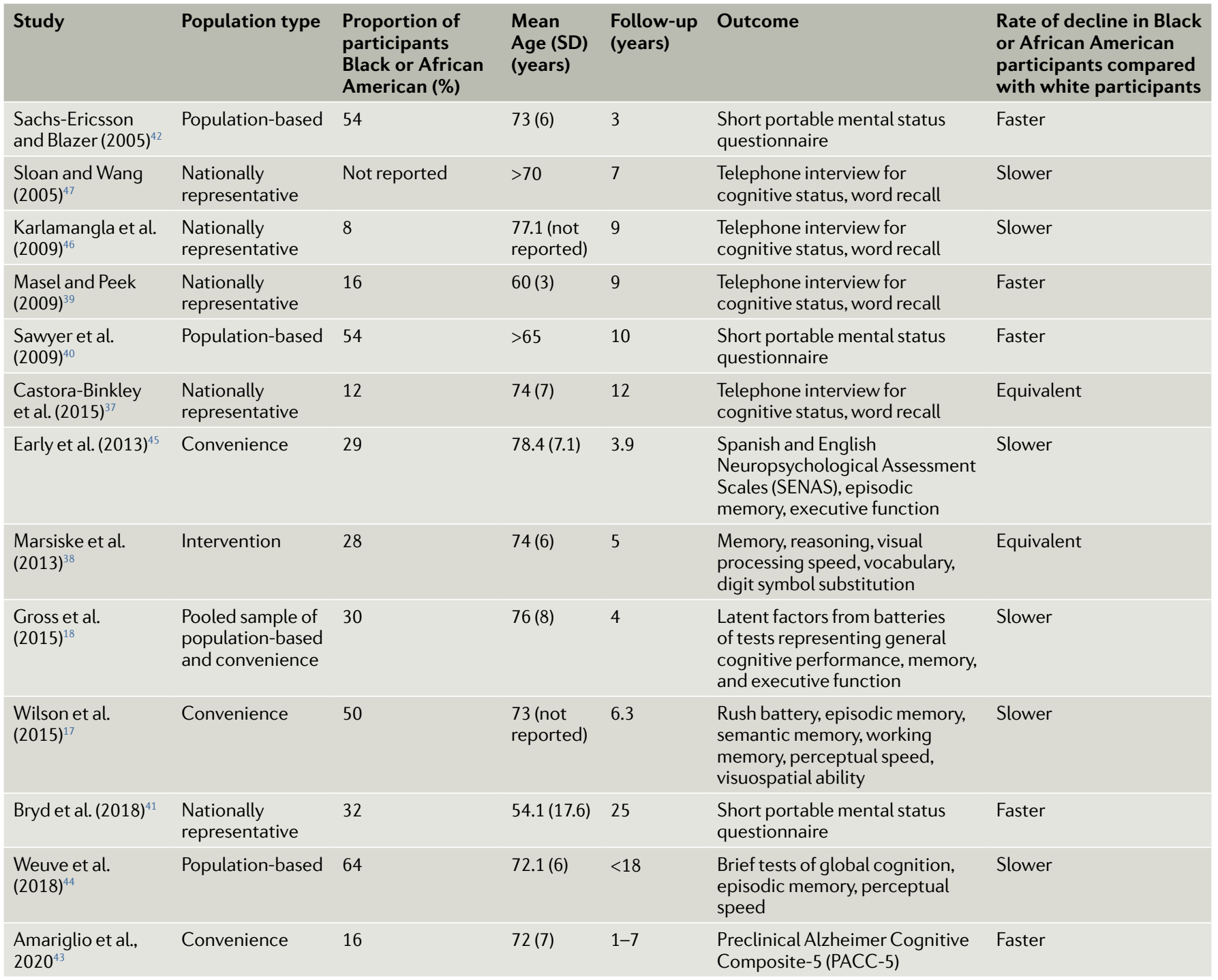

SD, standard deviation.

consistently found lower performance on neuropsychological tests by African American individuals than age-matched and education-matched white individuals, irrespective of dementia status s,16,22 $^{\text {. As }}$ highlighted above, a number of cultural and educational factors influence cognitive test performance and make the interpretation of racial differences in cross-sectional studies challenging. Although racial differences identified in cross-sectional studies could reflect stable differences in cognitive reserve, factors that influence cognitive reserve vary substantially across race ${ }^{34}$ and might differentially impact late-life cognition and potentially exacerbate disparities. However, studies that use repeated observations for each person over time overcome the inherent challenges associated with using cross-sectional designs to study racial differences in cognition, as the biases that affect a single test measurement are typically constant over time and are therefore less likely to affect analyses of individual changes in cognitive performance ${ }^{35}$. Thus, change in cognitive function over time is more meaningful than static observations of cognitive function when comparing African American individuals and white individuals. Measuring change in cognition over time is also a more realistic reflection of the actual course of $\mathrm{AD}$, which is characterized by progressive cognitive decline ${ }^{36}$.

In contrast to the results of cross-sectional studies, the results of longitudinal studies have been mixed. Some longitudinal studies in people without $\mathrm{AD}$ found equivalent rates of change in neuropsychological test performance in African American individuals and white individuals ${ }^{37,38}$, others found faster decline in cognitive ability among African
American individuals ${ }^{39-43}$, and still others found slower decline in African American individuals ${ }^{17,18,44-47}$. The factors contributing to these discrepant findings are uncertain, but are likely to have involved a combination of methodological issues related to recruitment and study engagement, sample selection, study design, measurement (for example, outcome measures with ceilings or with unequal interval scaling) and survival ${ }^{48-50}$. Taken together, the results of longitudinal studies suggest that there are probably not strong racial differences in trajectories of cognitive ageing $^{17}$. This observation is important because one might expect that the putative increased incidence of $\mathrm{AD}$ among African American individuals would be reflected in consistently faster rates of cognitive decline, the hallmark of a progressive neurodegenerative condition. 
Lack of data. The last important obstacle in interpreting racial differences in $\mathrm{AD}$, concerns the underlying pathology that causes dementia. Although neuropathological evaluation is still the gold standard for determining the presence and severity of common age-related pathologies in the post-mortem brain, few clinical-pathological studies have been conducted in older African American individuals. The majority of such studies used retrospective designs that included autopsies from medical examiners' cases, which came to autopsy regardless of the manner of death, and included little information on pre-mortem clinical or cognitive status ${ }^{51-54}$. One study found that $\mathrm{AD}$ and Parkinson disease-related pathology was more common in white individuals than African American individuals, whereas the prevalence of cerebrovascular disease was higher in African American individuals than in white individuals ${ }^{51}$. Other studies found no racial differences in the prevalence of AD pathology $y^{52,53,55,56}$. In one post-mortem study of brains from 13 African American individuals with a clinical diagnosis of $\mathrm{AD}$ who received a comprehensive clinical evaluation before death, a wide spectrum of vascular disease and $\mathrm{AD}$ pathology was observed - more than half of the participants had a mixture of $\mathrm{AD}$ pathology and stroke $\mathrm{F}^{57}$. In a sample of 122 participants with a diagnosis of clinical AD (41 African American individuals and 81 white individuals matched for age at death, sex, education, and cognitive status), mixed pathologies were far more common in African American individuals than white individuals ${ }^{58}$. Specifically, compared with white individuals, African American individuals with $\mathrm{AD}$ dementia were more likely to have AD pathology mixed with Lewy bodies or AD pathology mixed with
Lewy bodies and infarcts. On average, African American individuals also had more severe arteriolosclerosis and atherosclerosis than white individuals. These results have since been replicated in a much larger sample of African American individuals and white individuals from the National Alzheimer's Coordinating Center ${ }^{59}$.

Most of the neuropathological studies discussed here enrolled African American individuals with dementia from a clinic setting; however, the recruitment of study participants, particularly African American individuals, from a specialty memory clinic is known to be prone to selection bias $^{6,49}$. For example, because of fractured health-care relationships, mistrust and misconceptions about $\mathrm{AD}$ being a 'normal part of ageing' ${ }^{60}$, African American individuals are more likely to present to medical attention with behavioural problems, such as hallucinations, agitation and sleep disorders, than for memory problems ${ }^{6}$. Thus, longitudinal studies involving well-characterized community-dwelling African American participants who initially enrol without dementia need to be performed to examine racial differences in the underlying neuropathology of dementia. Interestingly, in such studies few racial differences in neuropathology have been observed ${ }^{61}$.

Similar to the lack of neuropathology data from African American individuals, few $\mathrm{AD}$ biomarker studies have been performed in this population and the results have been mixed. To date, all cerebrospinal fluid (CSF) AD biomarker studies, including those performed in participants without $\mathrm{AD}$ or mild cognitive impairment (MCI), have found lower levels of phosphorylated $\operatorname{tau}_{181}$ and total tau ${ }^{62-65}$ in African American individuals than in white individuals. However, studies of plasma and PET biomarkers in participants with and without $\mathrm{AD}$ found no difference in levels of tau between African American individuals and white individuals ${ }^{66-68}$. One study in people without dementia found higher amyloid PET burden in African American individuals than in white individuals ${ }^{69}$ (TABLE 2). The reasons for these discrepancies are unclear but the studies that measured tau in the CSF included relatively small samples of African American individuals and might have been prone to selection bias, given that African American individuals are less likely than white individuals to agree to lumbar puncture ${ }^{70}$. In addition, the extent to which results are influenced by genetics or disease status (for example, $\mathrm{MCI}$ or $\mathrm{AD}$ ) are unclear. Larger studies with representative samples of African American individuals with adjustment for the broad range of confounding factors are obviously needed.

\section{Strategies to move forward}

Notwithstanding the challenges in interpreting racial differences in $\mathrm{AD}$ and regardless of whether incidence of the disease is greater among African American individuals than white individuals the burden of AD faced by the African American community is undeniable and represents an urgent public health issue. Understanding this issue and developing solutions will not be possible without actively cultivating a new narrative of 'belonging' in research for a population that has been historically marginalized and disenfranchized. A number of important steps should be taken to achieve these goals.

Recruitment of study cohorts. Although $\mathrm{AD}$ is viewed as an urgent public health issue in the USA, resulting in widespread support for and focus on the development

\section{Table 2 | Studies of Alzheimer disease biomarkers in Black or African American individuals and white individuals}

\begin{tabular}{|c|c|c|c|c|}
\hline Study & Biomarker(s) & $\begin{array}{l}\text { Number of Black or } \\
\text { African American } \\
\text { participants }\end{array}$ & $\begin{array}{l}\text { Number of white } \\
\text { participants }\end{array}$ & $\begin{array}{l}\text { Biomarker levels in Black or } \\
\text { African American participants } \\
\text { compared with white participants }\end{array}$ \\
\hline Gottesman et al. $(2016)^{69}$ & A $\beta$ PET & 141 & 188 & Higher \\
\hline Garrett et al. $(2019)^{63}$ & CSF p-tau ${ }_{181}$ and t-tau & 152 & 210 & Lower \\
\hline Morris et al. (2019) ${ }^{64}$ & CSF p-tau ${ }_{181}$ and t-tau & 97 & 816 & Lower \\
\hline Meeker et al. $(2020)^{66}$ & $\begin{array}{l}\text { A } \beta \text { PET, tau PET and structural } \\
\text { and functional MRI }\end{array}$ & 70 & 434 & $\begin{array}{l}\text { No difference in tau PET, A } \beta \text { PET, or } \\
\text { functional MRI; lower brain volume }\end{array}$ \\
\hline Brickman et al. $(2021)^{67}$ & $\begin{array}{l}\text { Plasma } A \beta_{1-40}, A \beta_{1-42}, \text { t-tau, } \\
\text { p-tau }{ }_{181}, p-\text { tau }_{217} \text { and NfL; } A \beta \text { PET }^{a}\end{array}$ & 98 & 99 & No difference in any biomarker \\
\hline Rajan et al. $(2021)^{68}$ & Serum t-tau, NfL and GFAP & 811 & 516 & No difference in any biomarker \\
\hline
\end{tabular}

$A \beta$, amyloid- $\beta$, t-tau, total tau; $p$-tau, phosphorylated tau; NfL, neurofilament light chain; GFAP, glial fibrillary acidic protein. alncluded 100 Hispanic participants. 
of effective therapeutics, study cohorts are still shockingly and overwhelmingly homogeneous and white in terms of racial background. We can no longer afford to run research studies that do not reflect the diverse backgrounds and life experiences of the growing and increasingly diverse older adult US population. The scientific importance of enrolling people from under-represented populations in future studies is greater than the importance of enrolling people from groups already overrepresented in our studies. Furthermore, we will learn more about the disease if we enrol participants with different risk factors and life experiences, as opposed to continuing to enrol participants who are similar to those we have already studied. A sustained culturally sensitive outreach effort and ongoing community-wide education will be required to overcome the barriers that underlie low representation in research among African American individuals ${ }^{71}$. Such efforts will be essential for achieving successful recruitment and high rates of follow-up, and for encouraging participants to consent to invasive procedures such as, organ donation, lumbar puncture and PET that are often included in $\mathrm{AD}$ research. The recruitment of diverse samples that are representative of the community should be the goal of such efforts; differential selection into a study by race or ethnicity could give rise to selection bias and exacerbate disparities $^{49}$. Thus the study recruitment pipelines for African American individuals and white individuals must be intentionally designed so that they do not create an intractable selection bias.

Although examples exist of successful strategies for the recruitment and retention of participants from diverse backgrounds and life experiences ${ }^{13,50}$, the field is desperately in need of novel methods. Prior research has identified several successful approaches to overcoming barriers that deter African American individuals and other minoritized groups from participating in research. First, recruitment must be based on a foundation of mutual trust and respect, establishing a collaborative, mutually beneficial relationship between the community and the research team. The essence of this approach is to think of the community and future study participants as partners, as opposed to research subjects. Second, employing team members with extensive ties to the community and including sufficient numbers of staff and investigators who reflect the individuals in the community is helpful. Third, identifying key contacts within the African American community for networking purposes is important, recognizing that stakeholders are not always religious clergy or political figures. Fourth, recruitment and retention must be coupled with culturally sensitive community education that empowers people, providing not only factual knowledge about the disease but also healthy lifestyle recommendations to motivate brain health and wellness. Last, because diverse populations often have a high prevalence of other chronic health conditions beyond AD, providing ancillary services and incentives for participation can help sustain the long-term relationships that are important for longitudinal studies ${ }^{71,72}$.

In the last decade, innovative approaches that situate disparities within the historical inequities that caused them, while at the same time celebrating cultural assets, have also been shown to be effective strategies for increasing recruitment and engagement in research studies ${ }^{73-76}$. One study blended use of technology, neighbourhood walking and social reminiscence with the aim of maintaining cognitive health in older African American individuals with and without $\mathrm{MCI}^{74}$. Another study was a randomized trial within senior centres that evaluated the ability of a community choir programme called "The Community of Voices" to promote health and well-being ${ }^{77}$. Such approaches integrated with rigorous mixed methods can reveal unique participant perspectives about barriers to and facilitators of study participation, particularly for studies that require a higher level of commitment, such as brain autopsy ${ }^{78,79}$.

The work is challenging, the work is labour intensive, and the work is expensive, but the work has to be done for therapeutics and/or a cure to be effective in all. Creating an inclusive and diverse participant pool requires extensive efforts in building relationships, establishing trust and creating a culturally sensitive space that is transparent for participants and community partners. This space often needs to be created before recruitment begins to firmly situate the work within the broader contexts of peoples' lives and to align their interests and motivations with the goals of the research.

\section{Structural risk factors. The examination} of novel structural risk factors that are pertinent to the lived experience of older Black people ${ }^{80}$ and grounded in the broader discourse regarding their unique history within the USA ${ }^{81}$ is critical. For example, owing to institutionalized racism, neighbourhood racial residential segregation is an important societal characteristic that has caused a substantial proportion of the African American population to experience social environments where poverty and unemployment are the norm, where quality educational resources are lacking, and where social and physical deterioration make it difficult, if not impossible, to participate in behaviours that have been shown to protect brain health in late life ${ }^{82,83}$. High levels of neighbourhood-level social disadvantage and poverty, both common characteristics of most urban highly segregated cities, are associated with increased exposure to cardiovascular and metabolic health risk factors ${ }^{84,85}$, lower cognitive scores and greater neuropathology, independent of individual socioeconomic status ${ }^{86,87}$. A more comprehensive understanding of racespecific stressors, particularly at the broader socioenvironmental level, and how they 'get under the skin' to affect brain health, could lead to a deeper insight into the social mechanisms underlying health disparities. Such insights could ultimately facilitate the development of interventions, policies and improved therapeutics to address health disparities. Progress simply will not happen if we continue to link racial differences to biology or genetics instead of the common social forces that cause differences, including poverty, racism and inequitable educational resources.

Although racial disparities in $\mathrm{AD}$ are manifest during later life, it is becoming increasingly clear that they are often rooted in early-life exposures ${ }^{25,32}$. A number of seminal theories suggest that racial and ethnic disparities in old age are likely to reflect a cumulative effect of disadvantage across the life course ${ }^{88,89}$, and useful frameworks have been developed to foster investigation of structural and social determinants across different levels of analysis using a life course perspective ${ }^{90,91}$. Furthermore, because social determinants accumulate over time, the examination of risk factors from early life that shape opportunities and coping responses across the lifespan has the ability to uncover potentially novel factors that might have a role in the disease ${ }^{92}$. However, because of the inherent challenges involved in retrospective assessments of early life in older adults, studies that follow African American participants from young and mid-life to older age are needed. One example of such a study is Project Talent ${ }^{93}$, which is a national longitudinal study that first surveyed high school students in 1960 and now follows up with participants decades later to measure ageing outcomes. 


\section{Conclusion: we need more data}

A prevailing notion in the field is that African American individuals have a two to three times higher incidence of $\mathrm{AD}$ than white individuals. Although the burden of dementia is certainly high in the African American population, inconsistencies in the existing data and an important lack of data where it is most needed mean that we cannot be confident that the evidence supports this claim. Until and unless we change the narrative by increasing the number of studies that include African American participants, integrating risk factors that reflect the lived experience, and acquiring the biological data that will allow us to comprehensively examine the underlying mechanisms of disease, we will continue to have an incomplete understanding of racial differences in $\mathrm{AD}^{4}$. Importantly, we will come nowhere near developing therapeutics or at best, a cure, that will be effective in this population.

Lisa L. Barnes

Rush Alzheimer's Disease Center, Rush University Medical Center, Chicago, IL, USA.

e-mail: Lisa_L_Barnes@rush.edu

https://doi.org/10.1038/s41582-021-00589-3

Published online 6 December 2021

1. [No authors listed] 2020 Alzheimer's disease facts and figures. Alzheimers Dement. 16, 391-460 (2020).

2. Rovner, B. W., Casten, R. J. \& Harris, L. F. Cultura diversity and views on Alzheimer disease in older African Americans. Alzheimer Dis. Assoc. Disord. 27 133-137 (2013)

3. Mayeda, E. R., Glymour, M. M., Quesenberry, C. P. $\&$ Whitmer, R. A. Inequalities in dementia incidence between six racial and ethnic groups over 14 years. Alzheimers Dement. 12, 216-224 (2016).

4. Brewster, P. et al. Progress and future challenges in aging and diversity research in the United States. Alzheimers Dement. 15, 995-1003 (2019).

5. Rajan, K. B., Weuve, J., Barnes, L. L., Wilson, R. S. \& Evans, D. A. Prevalence and incidence of clinically diagnosed Alzheimer's disease dementia from 1994 to 2012 in a population study. Alzheimers Dement. 15, 1-7 (2019)

6. Barnes, L. L. \& Bennett, D. A. Alzheimer's disease in African Americans: risk factors and challenges for the future. Health Aff. 33, 580-586 (2014).

7. Chin, A. L., Negash, S., Xie, S., Arnold, S. E. \& Hamilton, R. Quality, and not just quantity, of education accounts for differences in psychometric performance between African Americans and white non-Hispanics with Alzheimer's disease. J. Int Neuropsychol. Soc. 18, 277-285 (2012).

8. Williams, D. R. Race and health: basic questions, emerging directions. Ann. Epidemiol. 7, 322-333 (1997).

9. Zuelsdorff, M., Barnes, L. L., \& Okonkwo, O. C. In Clinical Cultural Neuroscience: An Integrative Approach to Cross-Cultural Neuropsychology (ed. Pedraza, O.) 233-251 (Oxford Univ. Press, 2020).

10. Kunkle, B. W. et al. Novel Alzheimer disease risk loci and pathways in African American individuals using the African Genome Resources Panel: a meta-analysis. JAMA Neurol. 78, 102-113 (2021).

11. Hohman, T. J. et al. Global and local ancestry in African-Americans: implications for Alzheimer's disease risk. Alzheimers Dement. 12, 233-243 (2016).

12. Reitz, C. \& Mayeux, R. Genetics of Alzheimer's disease in Caribbean Hispanic and African American populations. Biol. Psychiatry 75, 534-541 (2014).

13. Babulal, G. M. et al. Perspectives on ethnic and racial disparities in Alzheimer's disease and related dementias: update and areas of immediate need. Alzheimers Dement. 15, 292-312 (2019).

14. Lind, K. E. et al. Effect of Medicare part D on ethnoracial disparities in antidementia medication use. J. Am. Geriatr. Soc. 66, 1760-1767 (2018).

15. Arvanitakis, Z., Shah, R. C. \& Bennett, D. A. Diagnosis and management of dementia: review. JAMA 322 , 1589-1599 (2019).

16. Zahodne, L. B., Manly, J. J., Azar, M., Brickman, A. M $\&$ Glymour, M. M. Racial disparities in cognitive performance in mid- and late adulthood: analyses of two cohort studies. J. Am. Geriatr. Soc. 64, 959-964 (2016).

17. Wilson, R. S., Capuano, A. W., Sytsma, J., Bennett, D. A $\&$ Barnes, L. L. Cognitive aging in older Black and White persons. Psychol. Aging 30, 279-285 (2015).

18. Gross, A. L. et al. Effects of education and race on cognitive decline: an integrative study of generalizability versus study-specific results. Psychol. Aging 30, 863-880 (2015).

19. Nelson, P. T. et al. Correlation of Alzheimer disease neuropathologic changes with cognitive status: review of the literature. J. Neuropathol. Exp. Neurol. 71, 362-381 (2012)

20. Campbell, A. L. et al. Caveats in the neuropsychological assessment of African Americans. J. Natl. Med. Assoc. 94, 591-601 (2002)

21. Wright, R. S. et al. Multiple influences on cognitive function among urban-dwelling African Americans. J. Racial Ethn. Health Disparities 6, 851-860 (2019).

22. Fyffe, D. C. et al. Explaining differences in episodic memory performance among older African Americans and Whites: the roles of factors related to cognitive reserve and test bias. J. Int. Neuropsychol. Soc. 17 , 625-638 (2011)

23. Kittner, S. J. et al. Methodological issues in screening for dementia: the problem of education adjustment. J. Chronic Dis. 39, 163-170 (1986).

24. Rivera Mindt, M., Byrd, D., Saez, P. \& Manly, J. Increasing culturally competent neuropsychological services for ethnic minority populations: a call to action. Clin. Neuropsychol. 24, 429-453 (2010).

25. Lamar, M. et al. Relationship of early-life residence and educational experience to level and change in cognitive functioning: results of the minority aging research study. J. Gerontol. B Psychol. Sci. Soc. Sci. 75, e81-e92 (2020)

26. Barnes, L. L. et al. Perceived discrimination and cognition in older African Americans. J. Int Neuropsychol. Soc. 18, 856-865 (2012).

27. Turner, A. D., James, B. D., Capuano, A. W. Aggarwal, N. T. \& Barnes, L. L. Perceived stress and cognitive decline in different cognitive domains in a cohort of older African Americans. Am. J. Geriatr. Psychiatry 25, 25-34 (2017).

28. Vonk, J. M. J. et al. Secular trends in cognitive trajectories of diverse older adults. Alzheimers Dement. 15, 1576-1587 (2019).

29. Zahodne, L. B., Sol, K. \& Kraal, Z. Psychosocial pathways to racial/ethnic inequalities in late-life memory trajectories. J. Gerontol. B Psychol. Sci. Soc. Sci. 74, 409-418 (2019).

30. Manly, J. J., Jacobs, D. M., Touradji, P., Small, S. A. $\varangle$ Stern, Y. Reading level attenuates differences in neuropsychological test performance between African American and White elders. J. Int. Neuropsychol. Soc. 8, 341-348 (2002).

31. Manly, J. J., Byrd, D. A., Touradji, P. \& Stern, Y. Acculturation, reading level, and neuropsychological test performance among African American elders. Appl. Neuropsychol. 11, 37-46 (2004).

32. Sisco, S. et al. The role of early-life educational quality and literacy in explaining racial disparities in cognition in late life. J. Gerontol. B Psychol. Sci. Soc. Sci. 70, 557-567 (2015)

33. Manly, J. J. Deconstructing race and ethnicity: implications for measurement of health outcomes. Med. Care. 44 (Suppl. 3), 10-16 (2006).

34. Avila, J. F. et al. Education differentially contributes to cognitive reserve across racial/ethnic groups. Alzheimers Dement. 17, 70-80 (2021).

35. Barnes, L. L. et al. Racial differences in the progression of cognitive decline in Alzheimer disease. Am. $J$. Geriatr. Psychiatry 13, 959-967 (2005).

36. Vos, S. J. B. et al. Preclinical Alzheimer's disease and its outcome: a longitudinal cohort study. Lancet Neurol. 12, 957-965 (2013).

37. Castora-Binkley, M., Peronto, C. L., Edwards, J. D. \& Small, B. J. A longitudinal analysis of the influence of race on cognitive performance. J. Gerontol. B Psychol. Sci. Soc. Sci. 70, 512-518 (2015).
38. Marsiske, M. et al. Race-related disparities in 5-year cognitive level and change in untrained ACTIVE participants. J. Aging Health 25 (Suppl.), 103-127 (2013).

39. Masel, M. C. \& Peek, M. K. Ethnic differences in cognitive function over time. Ann. Epidemiol. 19 778-783 (2009).

40. Sawyer, K., Sachs-Ericsson, N., Preacher, K. J. \& Blazer, D. G. Racial differences in the influence of the APOEepsilon 4 allele on cognitive decline in a sample of community-dwelling older adults. Gerontology 55, 32-40 (2009).

41. Byrd, D. R., Gee, G. C. \& Tarraf, W. Black-white mental status trajectories: what ages do differences emerge? SSM Popul. Health 6, 169-177 (2018).

42. Sachs-Ericsson, N. \& Blazer, D. G. Racial differences in cognitive decline in a sample of community dwelling older adults: the mediating role of education and literacy. Am. J. Geriatr. Psychiatry 13, 968-975 (2005).

43. Amariglio, R. E. et al. Examining cognitive decline across black and white participants in the Harvard Aging Brain Study. J. Alzheimers Dis. 75, 1437-1446 (2020).

44. Weuve, J. et al. Cognitive aging in black and white Americans: cognition, cognitive decline, and incidence of Alzheimer disease dementia. Epidemiology 29 151-159 (2018)

45. Early, D. R. et al. Demographic predictors of cognitive change in ethnically diverse older persons. Psychol. Aging 28, 633-645 (2013).

46. Karlamangla, A. S. et al. Trajectories of cognitive function in late life in the United States: demographic and socioeconomic predictors. Am. J. Epidemiol. 170 , 331-342 (2009)

47. Sloan, F. A. \& Wang, J. Disparities among older adults in measures of cognitive function by race or ethnicity. J. Gerontol. 60, 242-250 (2005).

48. Mayeda, E. R., Filshtein, T. J, Tripodis, Y, Glymour, M. M. \& Gross, A. L. Does selective survival before study enrolment attenuate estimated effects of education on rate of cognitive decline in older adults? A simulation approach for quantifying survival bias in life course epidemiology. Int. J. Epidemiol. 47, 1507-1517 (2018).

49. Gleason, C. E. et al. Association between enrollment factors and incident cognitive impairment in Blacks and Whites: data from the Alzheimer's Disease Center. Alzheimers Dement. 15, 1533-1545 (2019).

50. Gilmore-Bykovskyi, A. L. et al. Recruitment and retention of underrepresented populations in Alzheimer's disease research: a systematic review. Alzheimers Dement. 19, 751-770 (2019).

51. de la Monte, S. M., Hutchins, G. M. \& Moore, G. W. Racial differences in the etiology of dementia and frequency of Alzheimer lesions in the brain J. Natl. Med. Assoc. 81, 644-652 (1989).

52. Sandberg, G., Stewart, W., Smialek, J. \& Troncoso, J. C. The prevalence of the neuropathological lesions of Alzheimer's disease is independent of race and gender. Neurobiol. Aging 22, 169-175 (2001).

53. Miller, F. D., Hicks, S. P., D’Amato, C. J. \& Landis, J. R. A descriptive study of neuritic plaques and neurofibrillary tangles in an autopsy population. Am. J. Epidemiol. 120, 331-341 (1984).

54. Bonner, G. J., Darkwa, O. K. \& Gorelick, P. B. Autopsy recruitment program for African Americans. Alzheimer Dis. Dis. Assoc. Disord. 14, 202-208 (2000).

55. Wilkins, C. H., Grant, E. A., Schmitt, S. E., McKeel, D. W. \& Morris, J. C. The neuropathology of Alzheimer disease in African American and White individuals. Arch. Neurol. 63, 87-90 (2006).

56. Riudavets, M. A. et al. The prevalence of Alzheimer neuropathologic lesions is similar in blacks and whites. J. Neuropathol. Exp. Neurol. 65, 1143-1148 (2006).

57. Pytel, P. et al. Vascular and Alzheimer-type pathology in an autopsy study of African Americans. Neurology 66, 433-435 (2006)

58. Barnes, L. L. et al. Mixed pathology is more likely in black than white decedents with Alzheimer dementia. Neurology 85, 528-534 (2015)

59. Graff-Radford, N. R., Besser, L. M., Crook, J. E. Kukull, W. A. \& Dickson, D. W. Neuropathologic differences by race from the national Alzheimer's coordinating center. Alzheimers Dement. 12, 669-677 (2016).

60. Snowden, L. R. \& Graaf, G. COVID-19, social determinants past, present, and future, and African Americans' health. J. Racial Ethn. Health Disparities 8, 12-20 (2021)

61. Nag, S. et al. Limbic-predominant age-related TDP-43 encephalopathy in Black and White decedents. Neurology 95, e2056-e2064 (2020). 
62. Howell, J. C. et al. Race modifies the relationship between cognition and Alzheimer's disease cerebrospinal fluid biomarkers. Alzheimers Res. Ther. 9, 88 (2017).

63. Garrett, S. L. et al. Racial disparity in cerebrospinal fluid amyloid and tau biomarkers and associated cutoffs for mild cognitive impairment. JAMA Netw. Open 2, e1917363 (2019).

64. Morris, J. C. et al. Assessment of racial disparities in biomarkers for Alzheimer disease. JAMA Neurol. 76 264-273 (2019)

65. Kumar V V et al. Baseline results: the association between cardiovascular risk and preclinical Alzheimer's disease pathology (ASCEND) study. J. Alzhiemers Dis. 75, 109-117 (2020).

66. Meeker, K. L. et al. Socioeconomic status mediates racial differences seen using the AT(N) framework. Ann. Neurol. 89, 254-265 (2020).

67. Brickman, A. M. et al. Plasma p-tau181, p-tau217, and other blood-based Alzheimer's disease biomarkers in a multi-ethnic, community study. Alzheimers Dement 17, 1353-1364 (2021).

68. Rajan, K. B. et al. Remote blood biomarkers of longitudinal cognitive outcomes in a population study. Ann. Neurol. 88, 1065-1076 (2020).

69. Gottesman, R. F. et al. The ARIC-PET amyloid imaging study: brain amyloid differences by age race, sex, and APOE. Neurology 87, 473-480 (2016).

70. Blazel, M. M. et al. Factors associated with lumbar puncture participation in Alzheimer's disease research. J. Alzheimers Dis. 77, 1559-1567 (2020).

71. Denny, A. et al. Perspective on the "African American participation in Alzheimer disease research: Effective strategies" workshop, 2018. Alzheimers Dement. 16, 1734-1744 (2020)

72. Barnes, L. L. Shah, R. C. Aggarwal, N. T., Bennett, D. A. \& Schneider, J. A. The Minority Aging Research Study: ongoing efforts to obtain brain donation in African Americans without dementia. Curr. Alzheimer Res. 9, 734-745 (2012).

73. Robinson, R. A. S. et al. Framework for creating storytelling materials to promote African American/ Black adult enrollment in research on Alzheimer's disease and related disorders. Alzheimers Dement. 6 , e12076 (2020).

74. Croff, R. L. et al. Things are changing so fast: integrative technology for preserving cognitive health and community history. Gerontologist 59, 147-157 (2019).

75. Child, S. T. et al. 'We need a safe, walkable way to connect our sisters and brothers': a qualitative study of opportunities and challenges for neighborhoodbased physical activity among residents of low-income African-American communities. Ethn. Health 24, 353-364 (2019)

76. Green-Harris, G. et al. Addressing disparities in Alzheimer's disease and African-American participation in research: an asset-based community development approach. Front. Aging Neurosci. 11, 125 (2019).

77. Johnson, J. K. et al. Study protocol for a cluster randomized trial of the Community of Voices choir intervention to promote the health and well-being of diverse older adults. BMC Public. Health 15, 1049 (2015).

78. Glover, C. M., Shah, R. C., Bennett, D. A., Wilson, R. S $\&$ Barnes, L. L. The health equity through aging research and discussion (HEARD) study: a proposed two-phase sequential mixed-methods research design to understand barriers and facilitators of brain donation among diverse older adults. Exp. Aging Res. 46, 311-322 (2020)

79. Glover, C. M., Shah, R. C., Bennett, D. A., Wilson, R. S \& Barnes, L. L. Perceived impediments to completed brain autopsies among diverse older adults who have signed a uniform anatomical gift act for brain donation for clinical research. Ethn. Dis. 30, 709-718 (2020).

80. Thornton, R. L. et al. Evaluating strategies for reducing health disparities by addressing the social determinants of health. Health Aff. 35, 1416-1423 (2016).

81. Bailey, Z. D., Feldman, J. M. \& Bassett, M. T. How structural racism works - racist policies as a root cause of U.S. racial health inequities. N. Engl. J. Med. 384, 768-773 (2020).

82. Massey, D. S. \& Denton, N. A. American Apartheid: Segregation and the Making of the Underclass (Harvard Univ. Press, 1993).

83. Brown, S. C. et al. Neighborhood greenness and chronic health conditions in Medicare beneficiaries. Am. J. Prev. Med. 51, 78-89 (2016).

84. Pool, L. R. et al. Longitudinal associations of neighborhood-level racial residential segregation with obesity among blacks. Epidemiology 29, 207-214 (2018).
85. Kershaw, K. N. et al. Association of changes in neighborhood-level racial residential segregation with changes in blood pressure among black adults: the CARDIA study. JAMA Intern. Med. 177, 996-1002 (2017).

86. Hunt, J. F V et al. Association of neighborhood-level disadvantage with cerebral and hippocampal volume. JAMA Neurol. 77, 451-460 (2020).

87. Caunca, M. R. et al. Association of racial residential segregation throughout young adulthood and cognitive performance in middle-aged participants in the CARDIA study. JAMA Neurol. 77, 1000-1007 (2020).

88. Ferraro, K. F. \& Farmer, M. M. Double jeopardy, aging as leveler, or persistent health inequality? A longitudinal analysis of white and black Americans. J. Gerontol. B Psychol. Sci. Soc. Sci. 51, S319-S328 (1996).

89. Geronimus, A. T. Deep integration: letting the epigenome out of the bottle without losing sight of the structural origins of population health. Am. J. Public Health 103, S56-S63 (2013).

90. Jones, N. L. et al. Life course approaches to the causes of health disparities. Am. J. Public Health 109 S48-S55 (2019).

91. Hill, C. V., Pérez-Stable, E. J., Anderson, N. A. \& Bernard, M. A. The National Institute on Aging Health Disparities Research Framework. Ethn. Dis. 25, 245-254 (2015).

92. Barnes, L. L. et al. Effects of early-life adversity on cognitive decline in older African Americans and whites. Neurology 79, 2321-2327 (2012).

93. Chapman, B. P. et al. Association between high school personality phenotype and dementia 54 years later in results from a National US sample. JAMA Psychiatry 77, 148-154 (2020)

Competing interests

The author declares no competing interests.

Peer review information

Nature Reviews Neurology thanks the anonymous reviewers for their contribution to the peer review of this work.

\section{Publisher's note}

Springer Nature remains neutral with regard to jurisdictional claims in published maps and institutional affiliations.

(C) Springer Nature Limited 2021 